\title{
Funcionalidad y eficacia del posgrado. La visión del profesorado en la Universidad de Sonora
}

Manuel-Arturo Coronado-García, Sergio-Ramón Rossetti-López e Isaac-Shamir Rojas-Rodríguez

\begin{abstract}
RESUMEN
En este estudio se analiza la eficacia y funcionalidad del Posgrado en Administración de la Universidad de Sonora, de acuerdo con la percepción del personal docente de la misma. El interés de los posgrados en México sobre su nivel de eficacia y funcionalidad se ha incrementado, por ello es importante diagnosticar estos indicadores en la Universidad de Sonora, México. Se realizó un censo al personal docente del posgrado, donde se aplica un instrumento de medición basado en el modelo de Arturo de la Orden, donde el profesorado marcó el rango de eficacia o funcionalidad que consideran posee el Programa de Maestría en Administración. De acuerdo con el panorama de los resultados, en términos de porcentajes, se puede observar que la función de Preparación para el mundo profesional ocupa el primer lugar, con el $68.77 \%$ de cumplimiento de eficacia en ese sector, seguido de la función de Fomento y desarrollo de la ciencia, con un $63.10 \%$. El tercer lugar lo ocupa la función de Fomento, desarrollo y trasmisión de la cultura, con el $62.95 \%$. Con los resultados de esta investigación se emiten las recomendaciones correspondientes para una posible implementación de mejoras en un futuro cercano en materia.
\end{abstract}

Palabras clave: funcionalidad, eficacia, universidad, posgrado, Sonora, México.

Manuel-Arturo Coronado-García

\section{Sergio-Ramón Rossetti-López}

sergio.rossetti@unison.mx

Mexicano. Maestro en Gestión de Tecnologías de la Información, Universidad Tecmilenio, México. Profesor Tiempo Completo, Departamento de Administración, Universidad de Sonora, México. Temas de investigación: tecnologías de la información, tecnología educativa, gestión organizacional, ambientes virtuales de aprendizaje. ORCID: 0000-0002-5524-8674 
Funcionalidade e eficácia da Pós-graduação. A visão do professorado da Universidade de Sonora

\section{RESUMO}

Neste estudo se analisa a eficácia e funcionalidade da Pós-graduação em Administração da Universidade de Sonora, de acordo com a percepção da equipe docente da mesma. O interesse das pós-graduações no México sobre seu nível de eficácia e funcionalidade tem incrementado, por isso é importante diagnosticar estes indicadores na Universidade de Sonora. Se realizou um censo à equipe docentes da Pósgraduação, onde se aplica um instrumento de medição baseado no Modelo de Arturo De la Orden, onde o professorado marcou o rango de eficácia ou funcionalidade que consideram possuir o Programa de Mestrado em Administração. De acordo com o panorama dos resultados, em termos de porcentagens, podemos observar que a função de Preparação para o mundo profissional ocupa o primeiro lugar, com $68.77 \%$ de cumprimento de eficácia nesse setor, em segundo lugar a função de Fomento e desenvolvimento da ciência, com $63.10 \%$. O terceiro lugar foi ocupado a função de Fomento, desenvolvimento e transmissão da cultura, com $62.95 \%$. Com os resultados desta pesquisa se emitem as recomendações correspondentes para una possível implementação de melhoras em um futuro próximo.

Palavras chave: funcionalidade, eficácia, universidade, pós-graduação, Sonora, México.

\section{Functionality and efficiency of the Postgraduate courses. The vision of the teaching staff in the University of Sonora}

\section{ABSTRACT}

This study analyzes the effectiveness and functionality of the Graduate School of Management of the University of Sonora, according to the perception that the faculty has about it. The interest of postgraduate courses in Mexico about its level of effectiveness and functionality has increased, that is why the University of Sonora considered as a priority to diagnose these indicators. They carried out a survey to the postgraduate teaching staff, where a measurement instrument based on the Arturo de la Orden Hoz' s systemic model was applied. The teaching staff was asked to indicate the ranking of effectiveness or functionality that the Master's Program in Administration shows in their opinion. According to the results, it can be observed that the function of preparation for the professional world occupies the first place in terms of percentages, with $68.77 \%$ of effectiveness fulfillment in that sector, followed by the function of Promotion and development of science, with $63.10 \%$. The third place is occupied by the function of Promotion, development and transmission of culture, with $62.95 \%$. The results of this research made possible to issue the corresponding recommendations in order to reach in a near future significative improvements in this area.

Key words: functionality, efficiency, university, postgraduate, Sonora, Mexico. 


\section{Introducción}

En la actualidad la educación superior y de posgrado ha tenido un auge notorio en todo el mundo. En este sentido, Didriksson (2008) señala que de acuerdo con la Organización de Naciones Unidas (ONU), en 1950 existían 75 universidades en América latina, y en 2018 hay alrededor de 1500. Este incremento es concebido como una reacción de los gobiernos para elevar el nivel de vida de los habitantes; contribuyendo por consiguiente al desarrollo regional. Por lo tanto, las universidades y los posgrados se han convertido en actores importantes dentro de la formación de individuos para la generación de mayores impactos hacia la sociedad.

En el caso concreto de este estudio, se busca medir la pertinencia social mediante las variables de funcionalidad y eficacia del Programa de Posgrado en Administración de la Universidad de Sonora (UNISON), a través de la visión del profesorado. Los enfoques sobre los cuales los sujetos del referente emitirán sus percepciones serán, por un lado, la funcionalidad, considerando un modelo de funcionalidad aplicado en universidades, y por otro lado, el grado de eficacia, de acuerdo con la percepción de los sujetos entrevistados a lo largo del estudio. Se ha elegido a los docentes principalmente porque son ellos quienes desarrollan gran parte de las funciones universitarias en su área de adscripción, por ende, pueden emitir un valor acerca del cumplimiento de dichas funciones, pero sobre todo de las áreas de oportunidad para alcanzar los objetivos planteados por su departamento de adscripción.

Este proyecto de investigación forma parte de la búsqueda de nuevos modelos para medir pertinencia y funcionalidad institucional, por un lado de educación y formación universitaria, y por otro, de administración y gestión organizacional. En esencia, este estudio se centra en valorar el cumplimiento funcional del Posgrado en Administración y su alineación con el entorno actual, basado en un modelo aplicado en diferentes contextos y referentes empíricos de carácter universitario.
Este análisis cifra su importancia en que las inercias y dinámicas de los sectores externos al medio educativo, resaltan la necesidad de transformar y evolucionar los procesos de formación de quienes egresan del posgrado. Por tanto, puede ser importante la generación de pautas para una revolución del pensamiento de quienes intervienen en estos procesos de formación, y así contribuyan al ajuste y alcance de la calidad exigida en los programas, así como a la satisfacción de los requerimientos del entorno profesional de la actualidad. De igual manera, este trabajo pretende reforzar las bases para valorar el cumplimiento de la pertinencia social del posgrado como atributo que refleja los resultados y efectos de los procesos formativos que en él se imparten.

El propósito de este estudio es valorar el cumplimiento de las funciones e impacto del Posgrado en Administración de la UNISON, a través de un modelo de funcionalidad universitaria (De la Orden, 2007), desde la perspectiva de los profesores del programa, ya que son quienes llevan a cabo las funciones de formación y aplicación de los métodos de transmisión de la información educativa, fruto de la implementación de los conocimientos en los estudiantes. El presente estudio se basa en la aplicación específica de un modelo de Funcionalidad y Eficacia (De la Orden 2007), por lo que los resultados sólo reflejarán el comportamiento de la información generada en relación con dicho modelo. De igual manera, solamente se contempla aplicar instrumentos a los profesores del posgrado mencionado. El periodo temporal de la investigación fue de agosto a diciembre de 2017.

\section{Referente teórico}

Durante el desarrollo del presente análisis se trabajaron principalmente tres categorías analíticas o conceptos fundamentales. El primero es la funcionalidad, que se refiere al grado en que una organización plantea y cumple sus funciones, desde el punto de vista de autores como De la Orden (2007). En segunda 
instancia, otro concepto importante para el sustento de esta investigación será la eficacia organizacional, la cual nos remonta a los principios administrativos de Fayol (Claude, 2005). Y por último, el posgrado, que durante su existencia ha sido estudiado y abordado, sustantiva y adjetivamente por varios autores (entre ellos, García 2009). Estas categorías son de importancia para el estudio; el enfoque de funcionalidad deriva de la ciencia administrativa, la que desde la escuela de la administración científica, ha buscado alcanzar los objetivos planteados por las organizaciones.

Para entrar en materia pudiese resultar relevante definir organización y eficacia para conceptualizar los ejes que sustentan la investigación. De acuerdo con el Diccionario de la Real Academia Española, la palabra organización viene del griego organón que quiere decir "instrumento", "herramienta" o "útil”; así, el término organización alude al cúmulo o grupo de personas que se unen con un fin determinado, aunque también puede referirse a una entidad, empresa, institución pública, organización no gubernamental o corporación, entre otros, que son creadas por personas para una función en particular, por ende se manifiesta que el concepto de organización puede aplicarse en diferentes ámbitos, tales como el educativo, el empresarial, religioso, deportivo, entre muchos otros. Además, se define organización como "asociación de personas regulada por un conjunto de normas en función de fines determinados" (Koont'z y Weihrich, 1988).

De acuerdo con lo anterior, la organización comprende la identificación y clasificación de las actividades requeridas, el agrupamiento de las actividades necesarias para lograr los objetivos, la asignación de cada agrupamiento a un administrador con la autoridad necesaria para supervisarlo (delegación). De igual manera, genera las medidas para coordinar horizontalmente (en el mismo nivel organizacional o en uno similar) y verticalmente (por ejemplo, las oficinas centrales corporativas, la división y el departamento en la estructura organizacional) (Koont'z y Weihrich, 1988).

Por otra parte, Stephen Robins y Coulter (2005) señalan que la eficacia es hacer lo que es apropiado, es decir, las actividades de trabajo que ayudan a la organización a alcanzar sus metas. Por su parte, Chiavenato (2014) indica que la eficacia es una medida normativa del logro de los resultados. Puede medirse en función de los objetivos logrados. También se refiere a la capacidad de una organización de satisfacer una necesidad social mediante el suministro de bienes y servicios.

Una vez definidos los conceptos de eficacia y organización, se puede asumir que la eficacia organizacional es punto clave para cualquier ente que se dedique a obtener resultados medibles en el cumplimiento de sus metas y el Posgrado en Administración es parte de ello. Cuando se manejan los conceptos de funcionalidad, eficacia y eficiencia se hace referencia a los modelos y enfoques administrativos y empresariales. Estos conceptos nos remontan a los principios del proceso administrativo de Taylor y Fayol (Claude, 2005), que "nos hacen ver que para que una organización pueda ser funcional requiere hacer las cosas bien y con la maximización de sus recursos, dicho de otra manera, ser eficaz y eficiente" (Coronado, 2017: 23).

Por otro lado, De la Orden (2007) abordó la funcionalidad como grado de cumplimiento de las funciones, enfocado a las universidades, además, analizó hasta qué punto este cumplimiento satisface las expectativas de sus usuarios. La tabla 1 muestra las funciones de De la Orden (2007), que ya han sido abordadas en estudios de funcionalidad organizacional en universidades (Coronado, 2017).

Este modelo refleja los puntos Funcionalidad y Eficacia organizacional, los cuales serán analizados en la Maestría en Administración. La eficacia en una organización se refiere al cumplimiento de las tareas y funciones, es decir, concluir una tarea, meta, objetivo, proyecto etcétera. La pertinencia de 
utilizar este modelo recae en la naturalidad de las funciones universitarias, es decir, las funciones propuestas por De la Orden (2007) y Coronado (2017), son las que generalmente se llevan a cabo en las universidades, aunque son presentadas éstas con mayor especificidad, es decir, no se engloban en la docencia, investigación y extensión, sino divididas en aquellas donde, sin dejar de cumplir estas funciones clásicas, se persigue una mayor eficacia institucional, pero sobre todo un mayor impacto hacia la sociedad. También, si con los recursos otorgados se anotan más logros de los propuestos, se es igualmente eficiente. La eficacia se plasma en alcanzar la mayor cantidad de metas y objetivos o cumplimiento de indicadores (Valenzuela y Ramírez, 2011) posibles trazados, independientemente de los recursos, aunque siguen siendo importantes. Pero en el sentido propio de la palabra podemos diferenciar eficacia de eficiencia, la primera es el logro de objetivos y metas y la segunda maximización de recursos; aunque ambas siguen íntimamente relacionadas para el mejor manejo de una organización.

Tabla 1. Categoría de las funciones universitarias

\begin{tabular}{|c|c|}
\hline Funciones & Finalidades \\
\hline Fomento y desarrollo de la ciencia & $\begin{array}{l}\text { - Fomento y desarrollo de la investigación } \\
\text { - Científica } \\
\text { - Formación de científicos } \\
\text { - Difusión e intercambio de avances científicos }\end{array}$ \\
\hline Fomento y desarrollo de la tecnología & $\begin{array}{l}\text { - Fomento y desarrollo de nuevas tecnologías } \\
\text { - Formación de tecnólogos y técnicos de alto nivel } \\
\text { - Difusión e intercambio de avances tecnológicos }\end{array}$ \\
\hline Fomento y desarrollo de la actividad económica & $\begin{array}{l}\text { - Fomento y desarrollo de la actividad económica } \\
\text { - Formación de emprendedores } \\
\text { - Difusión de alternativas para la actividad económica }\end{array}$ \\
\hline Fomento, desarrollo y transmisión de la cultura & $\begin{array}{l}\text { - Fomento, desarrollo y difusión de la cultura } \\
\text { - Formación inicial y continuada para el goce estético y el arte } \\
\text { - Difusión e intercambio de producciones culturales }\end{array}$ \\
\hline $\begin{array}{l}\text { Preparación para el mundo profesional o } \\
\text { profesionalización }\end{array}$ & $\begin{array}{l}\text { - Profesionalización inicial y continuada para las tareas sociales que exijan un } \\
\text { conocimiento científico } \\
\text { - Formación genérica que promueva la participación activa en sociedad } \\
\text { - Difusión de valores éticos y culturales de la profesionalidad }\end{array}$ \\
\hline Compromiso Social & $\begin{array}{l}\text { - Fomento de actitudes de contribución al progreso y al mantenimiento de la } \\
\text { calidad de vida y responsabilidad social } \\
\text { - Formación social } \\
\text { - Difusión e intercambio de responsabilidad social y valores universales }\end{array}$ \\
\hline
\end{tabular}

Fuente: categorización de De la Orden (2007). 
Estos conceptos remontan a los principios del proceso administrativo de Taylor y Fayol (Claude, 2005), y recuerdan que para que una organización pueda ser funcional requiere de hacer las cosas bien y con la maximización de sus recursos, dicho de otra manera, ser eficaz y eficiente. En relación con este análisis, De la Orden (2007) ve la funcionalidad como grado de cumplimiento de las funciones, enfocado a las universidades, pero además hasta qué punto este cumplimiento satisface las expectativas de sus usuarios. De acuerdo con esto aparecen los aspectos de pertinencia en la formación universitaria, que además de cumplirse a través de las funciones, también se pueden valorar a través de las competencias de los estudiantes y desde la perspectiva de comprobar su interdependencia en conjunto con calidad y funcionalidad, muy probablemente por la misma responsabilidad que corren las instituciones de educación superior (IES, ver figura 1). De igual manera, otros autores como Fisher, Dornbush y Schmalensee (2004), hacen un análisis costo beneficio en la educación, y señalan que es difícil medir la eficacia o eficiencia de este sistema, ya que hay un desfase entre los tiempos de las erogaciones y de los beneficios. Por lo tanto, medir la eficacia de la universidad sólo se puede proyectar a largo plazo en la educación por lo que la funcionalidad suele presentar diversos escenarios.

\section{Figura 1. Modelo sistémico de la educación universitaria}

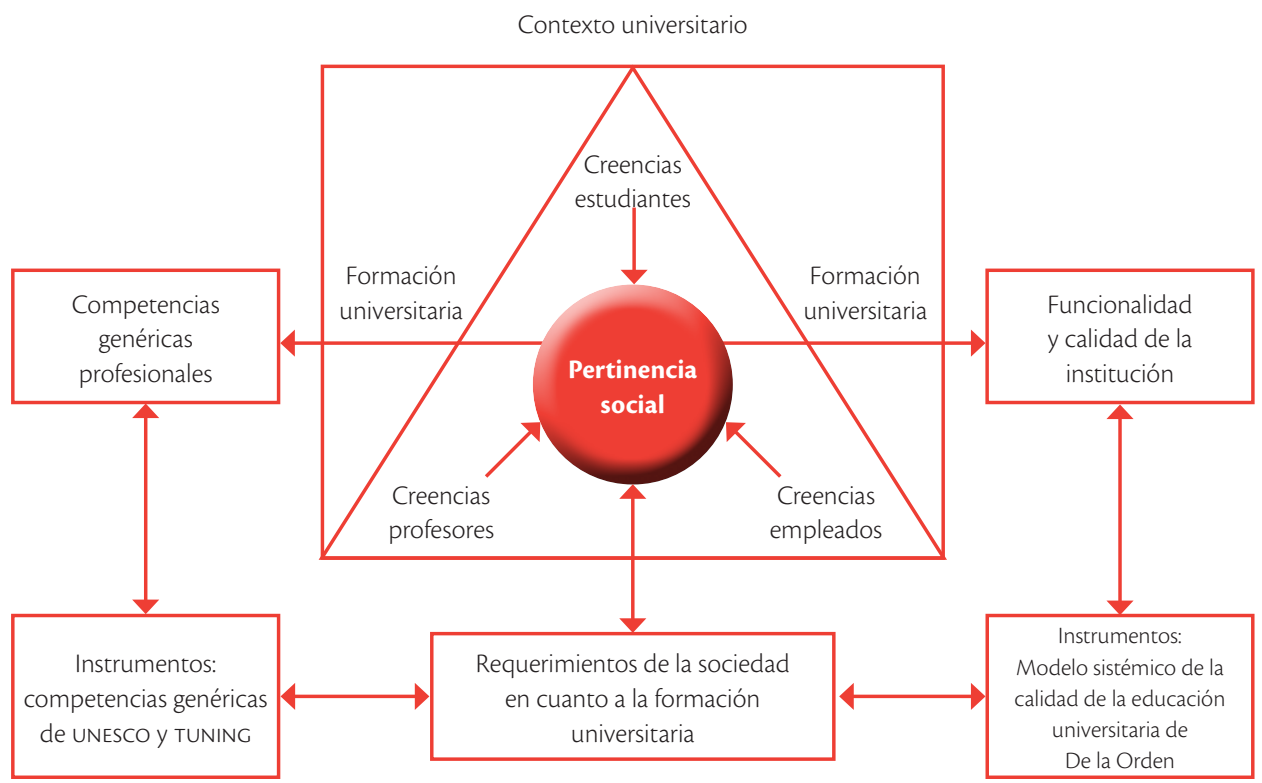

Fuente: aplicación de Modelos de De la Orden (2007) y del Tuning Project (2007), en Coronado (2017). ${ }^{1}$

\footnotetext{
${ }^{1}$ Modelo de percepción de pertinencia social y de la transmisión, adquisición y valoración de competencias genéricas, mediante la formación universitaria. Obtenido del libro Pertinencia Social Universitaria, enfoques y perspectivas de profesores estudiantes y empleadores, Hermosillo, México, Editorial Qartuppi, DOI: 10.29410/QTP.17.03.
} 
No obstante lo anterior, es interesante medir resultados a corto plazo, y esto sí puede realizarse dentro del sistema educativo mexicano, sobre todo en los niveles altos, quienes responden por los resultados del sistema y deben expresarlos constantemente. Uno de los niveles más importantes para este efecto de la educación en México es la supervisión. La Organización para la Cooperación y el Desarrollo Económicos (OCDE) en sus estudios económicos del 2005 en México, evidenció que el sistema educativo mexicano no muestra un desempeño suficientemente adecuado para reducir el rezago educativo en un tiempo apropiado.

Si se realizara un análisis sobre estos elementos, se podría señalar que hasta 2005 el sistema educativo mexicano no era del todo eficiente, debido a los altos índices de deserción durante los primeros años de las cohortes en nivel superior, y los bajos rendimientos en cuanto a estándares internacionales. Al respecto, políticamente se propuso que las instituciones de educación debían certificarse para lograr la eficiencia adecuada, sin embargo, con el correr del tiempo se pudo percibir que las certificaciones no garantizan la mejora del desempeño del sistema educativo nacional. Probablemente México aún no estaba listo para adoptar una cultura de evaluación o certificación, lo importante, y sobre todo apegado a la realidad, es que hoy es necesario estar en esa dinámica actual de los procesos de educación, emanados de los resultados de la interrelación global en materia de educación y eficacia institucional.

En resumen, se puede analizar la funcionalidad institucional desde un punto de vista social. Por tanto, sería interesante definir claramente las necesidades de administración funcional de las instituciones y del sistema educativo y ver si existe la capacidad, por parte de los perfiles de supervisión, de obtener mejores resultados. $\mathrm{Al}$ respecto se puede retomar la cuantificación de los beneficios sociales de la educación, para así poder medir con mayor exactitud el desempeño de quienes laboramos en un ámbito social como la educación.

\section{Metodología}

En este estudio se hace referencia en mayor medida a la eficacia de un departamento de posgrado como organización funcional, estableciendo como parámetro lo que la sociedad espera de la misma, todo esto visto a través de sus profesores.

Por otra parte, los parámetros de funcionalidad son aplicables casi de manera natural a instituciones como las universidades, basados en valores, aspiraciones y necesidades generales, con ausencia de acuerdo sobre las metas organizacionales deseables. En todo caso, existen técnicas para determinar los niveles de aceptación de los valores, metas y productos de las instituciones universitarias (De la Orden, 2007). En el caso del posgrado que nos ocupa, la base principal son los indicadores de funcionalidad.

Estos índices pueden agruparse en cuatro categorías, en primer lugar, las que expresan relaciones entre las entradas al sistema universitario y los valores sociales: equidad en el sistema de reclutamiento de alumnos y profesores, y equidad de acceso; verificar que no exista alguna barrera que se oponga a este principio; equidad de asignación de recursos (entrando un poco en materia de eficiencia, es importante como se van a asignar los recursos para alcanzar los objetivos), y demanda de inscripción.

La segunda categoría expresa relaciones entre estructura, procesos directivos y de gestión en las instituciones universitarias, así como los valores, expectativas y necesidades sociales: estructura de autoridad; clima organizacional; validez cultural, social y laboral del currículum y de los programas académicos; validez curricular del sistema de evaluación; etcétera.

La tercera contiene elementos que expresan relaciones entre producto y resultados de los programas académicos, así como expectativas y necesidades generales: adecuación en cantidad y modalidad de los graduados; vigencia de conocimientos, aptitudes y competencias adquiridos; relevancia de valores y actitudes; relevancia de la aportación científica investigadora, etcétera. 
Por último, la cuarta categoría expresa relaciones entre metas y objetivos de la educación universitaria, así como las aspiraciones, expectativas y demandas de formación superior.

De acuerdo con lo anterior, la eficacia y eficiencia son necesarias para la mayoría de las organizaciones del mundo, y desde hace ya algunos años, cuando se habla de funcionalidad, eficacia y eficiencia se hace referencia a los modelos y enfoques administrativos y empresariales.

\section{Enfoque y alcances}

Para llevar a cabo esta investigación, fue necesario delimitar los sujetos a investigar y, apegados al objetivo, determinar la ruta para atender al planteamiento y objetivo de la investigación. Una vez enmarcada la investigación se determina que el estudio es de tipo descriptivo, con enfoque mixto, mayormente cuantitativo, sin ser analítico ni inferencial. Esto debido a que se manejaron datos numéricos para la obtención de medias, acordes al modelo utilizado. De igual manera, aunque con menor incidencia, se analizaron datos cualitativos de los sujetos investigados.

\section{Población de estudio}

Las fuentes de información utilizadas fueron primarias y secundarias. Las fuentes primarias fueron los cuestionarios aplicados a los profesores del Posgrado en Administración de la Universidad de Sonora. Las fuentes secundarias que se utilizaron mayormente fueron documentos para el sustento de información numérica y cualitativa, de algunas instituciones como la Asociación Nacional de Universidades e Instituciones de Educación Superior (ANUIES), Secretaría de Educación Pública (SEP) y la UNISON. Debido al número de profesores adscritos al Departamento de Administración, se decidió hacer un censo para la aplicación de instrumentos, generando con ello un total de 13 cuestionarios contestados para su análisis.

\section{Técnicas de recolección}

Para recolectar la información se utilizaron cuestionarios, los cuales se aplicaron en reuniones de manera directa y personal con cada uno de los sujetos participantes. El cuestionario que se utilizó está dividido en dos partes, la primera, para la información general de los sujetos analizados; la segunda, para la valoración de las características del modelo de funcionalidad y pertinencia de De la Orden (2007). Los ítems están estructurados en diferentes categorías, con opciones de respuestas en escala de Likert del 1 al 7, siendo 1 el de menor valor y 7 el de mayor valor.

\section{Procesamiento de información}

Para el análisis y procesamiento de la información, se recurrió al manejo validado por los modelos utilizados, teniendo como base el uso de hoja de cálculo Excel, dado que el estudio consistió en un análisis de medias de resultados, generando la valoración integral sobre la funcionalidad y eficacia del Posgrado. Por último, se clasificaron categorías de análisis para encaminarnos a responder de forma sistémica el grado de cumplimiento de los aspectos ya analizados por parte del programa. Estas categorías comprenden tanto a sujetos como variables complementarias que de forma individual y conjunta brinden una perspectiva integral de los hallazgos para su valoración y conclusión. Los instrumentos que se utilizarán han sido ya validados en otros estudios de similar índole en diversas partes del mundo, y cuyo sustento ha sido abordado con anterioridad.

\section{Resultados}

Una vez procesada la información, se generaron los resultados conforme a las categorías enunciadas en cada uno de los parámetros de funcionalidad del modelo empleado. Para ello, primeramente se describieron las características de los sujetos entrevistados, en este caso, los profesores del Posgrado en Administración. 
Como puede observarse en la tabla 2, la mayoría de los maestros censados son casados con un promedio de edad superior a 60 años. De igual manera, la mayor parte de los encuestados cuenta con un promedio de años de experiencia en docencia por encima de los 30 años. El campo de estudio de experiencia sin docencia no es tan alto, 17 años en promedio, pero aun así es una cantidad de experiencia más que considerable en cualquier campo laboral, tanto público como privado. Cabe destacar también que los docentes cuentan con un promedio de 26 años de antigüedad en la institución.

En su carga académica, la mayoría cuenta con dos asignaturas, la minoría tiene tres y un caso aislado con una sola materia. En cuanto al nivel educativo o grado de habilitación de los entrevistados, todos poseen licenciatura, sólo unos cuantos estudiaron una especialidad, el 100\% cuenta con nivel Maestría y un poco más de la mitad cuenta con estudios de doctorado.

Con esta información se puede señalar que el Posgrado en Administración cuenta con un personal docente altamente calificado, y con un umbral de experiencia amplio en diversos sentidos, más aún en las áreas económico-administrativas. Este punto es relevante para validar las respuestas esperadas por el instrumento y el modelo de funcionalidad universitaria.

Tabla 2. Características de los sujetos entrevistados (docentes del Posgrado en Administración de la UNISON)

\begin{tabular}{|c|c|c|c|}
\hline \multicolumn{3}{|c|}{ Características de los docentes de Maestría en Administración unISON en el estudio 2017} & \\
\hline Exp. laboral con docencia & Entre 17-40 años de experiencia & 31 años & promedio \\
\hline Exp. laboral sin docencia & Entre $0-40$ años & 17 años & promedio \\
\hline Disciplina & 84.61\% ciencias económico-administrativas & $15.38 \%$ otras disciplinas & \\
\hline Cuerpo académico (CA) & 5 Maestros en CA & $8 \sin$ CA académico & \\
\hline lic/ing & ING/4 & $\mathrm{LIC/7}$ & $\mathrm{CP} / 2$ \\
\hline Especialidad & $15 \%$ Docentes & & \\
\hline
\end{tabular}

Fuente: categorización de De la Orden (2007). 
En otro sentido, se puede aseverar que, ante la poca rotación de la planta docente, se cuenta con grupos interdisciplinares consolidados e incluso cuerpos académicos reconocidos por su trabajo, y por el de cada uno de los miembros. Para una universidad esto es importante para la generación, aplicación y transmisión de conocimiento y experiencia, urgente para las nuevas generaciones de estudiantes, y para la sociedad en general. En cuanto al tema de la investigación, se visualiza difícil una organización eficaz y funcional sin el capital humano requerido, por lo que, en este caso, los profesores manifiestan tener los atributos para coadyuvar en la funcionalidad de su departamento. Una vez concluida la descripción de los profesores participantes en la investigación, se generaron los resultados de acuerdo con los reactivos de cada una de las funciones y del grado de eficacia percibida.
Pueden apreciarse en la gráfica 1, los puntos más fuertes de la función desarrollo de la ciencia: las preguntas P14 (5.86), P12 (5.71), P1 1 (5.61) y P13 (5.5), en ese orden concernientes a los métodos propios de los docentes y su enseñanza; evaluaciones que practican en sus materias; coherencia del programa, y proporción teórico-práctica de éste. Mientras que los puntos más débiles de esta área se encuentran en las preguntas 25(2.51), P16 (3.38), P17 (3.55), P6 (4), concernientes a avances de investigación del posgrado en administración, trascendencia de la frontera de los mismos; procesos de autoevaluación de la maestría, y la medida en que los recursos colaboran con los fines y objetivos.

Por otro lado, en cuanto a la función de fomento y desarrollo de la tecnología, aunque el Posgrado en Administración no es del ámbito tecnológico, no es ajeno a su importancia, y el instrumento arrojó los resultados ques se aprecian en la gráfica 2.

\section{Gráfica 1. Resultados de la función de fomento y desarrollo de la ciencia}

Función 1. Desarrollo de la ciencia. Puntuación promedio

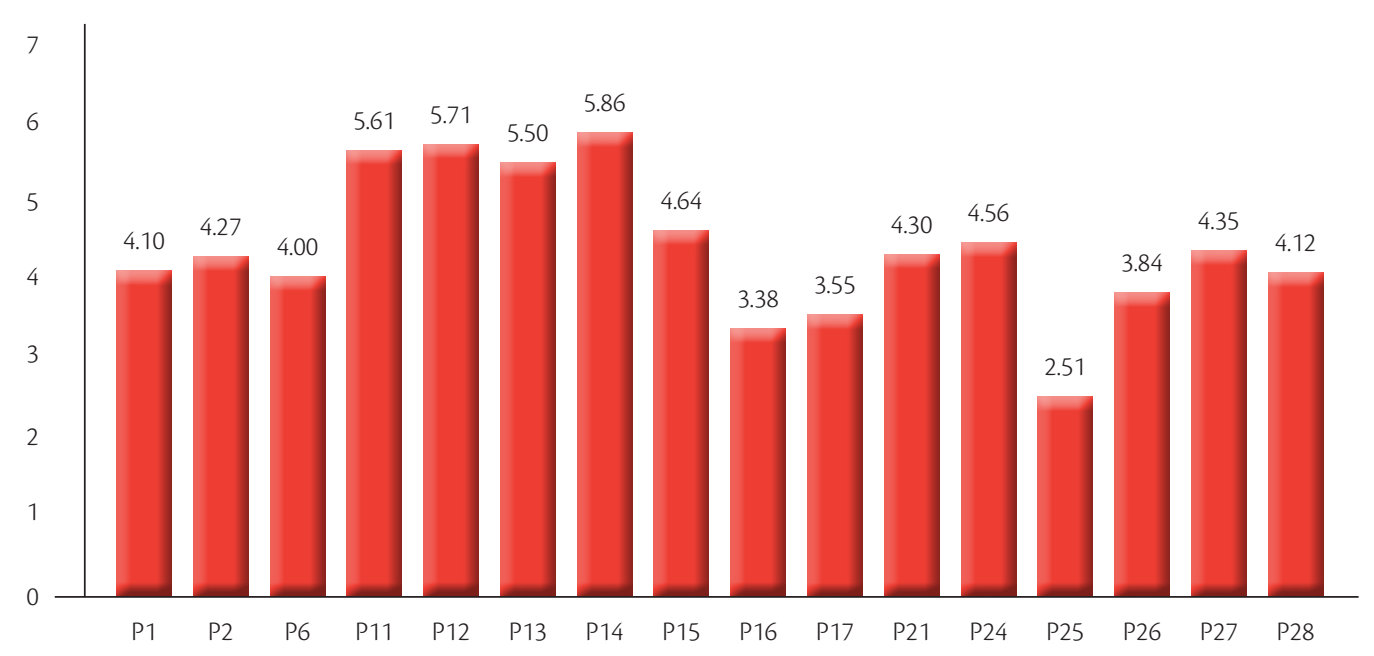

Fuente: elaboración propia de acuerdo con los resultados generados con la investigación. 


\section{Gráfica 2. Resultados de la función de fomento y desarrollo de la tecnología}

Función 2. Desarrollo de la tecnología. Puntuación promedio

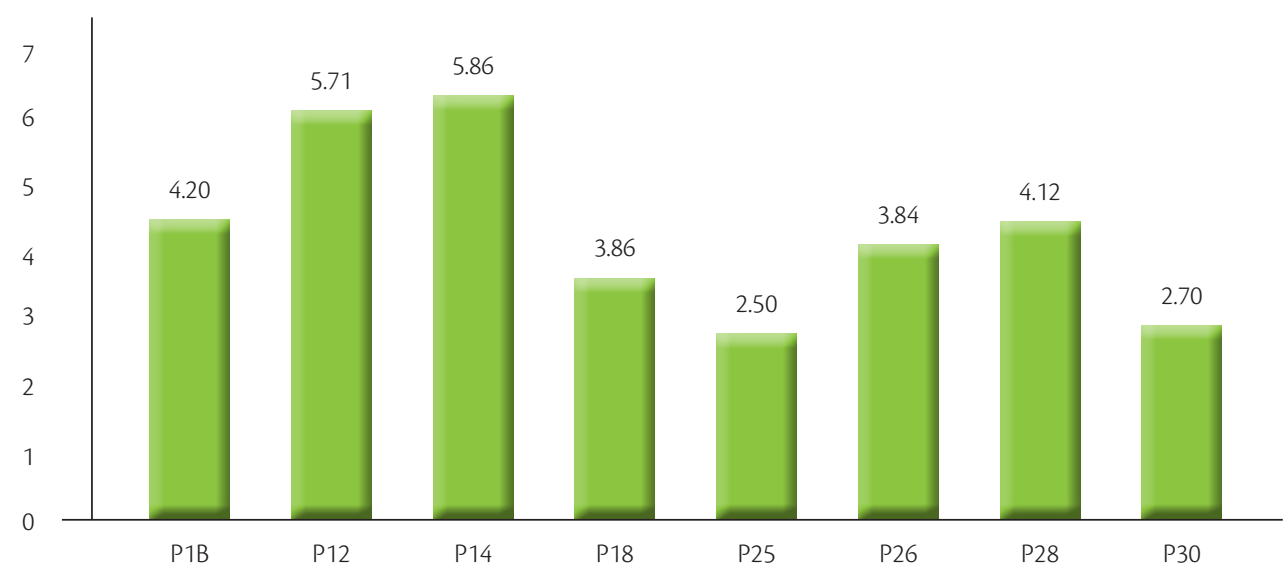

Fuente: elaboración propia de acuerdo con los resultados generados con la investigación.

En la función del desarrollo de la tecnología se pueden apreciar los puntos que sobresalen por encima del resto, correspondientes a las preguntas P14 (5.86) y P12 (5.71), referidas a las características propias de enseñanza de los profesores y las evaluaciones que aplican a los estudiantes.

En cuanto a los puntos frágiles en esta función, relativos a las preguntas P25 (2.5) y P30 (2.7), éstos están relacionados con la investigación propia, la generación de patentes, y diversos avances tecnológicos, o bien teóricos de los cuales adolece la Maestría en Administración, según la percepción de los profesores.

También las experiencias de intercambio con otros centros de investigación para docentes y posgraduados son escasas. En los demás puntos, se mantiene una posición neutral en cuanto a la tecnología.

Pasando a la siguiente función, fomento y desarrollo de la actividad económica, cabe aclarar que si bien no es una función del posgrado, si debiera intervenir activamente en los procesos de planeación, ejecución y evaluación de los procesos económicos (De la orden, 2007); en cierta medida esto ocurre, sin embargo, de acuerdo con la percepción de los profesores, existen aún áreas de oportunidad al respecto. De igual manera se reflejan algunos aspectos positivos, anotados en la gráfica 3. En esta gráfica se observan dos polaridades marcadas entre un punto sobresaliente y otro demasiado bajo, acompañado por una marcada neutralidad en los demás reactivos. El punto fuerte corresponde a la pregunta: $\mathrm{P} 1 \mathrm{C}$ (4.7), correspondiente al fomento y desarrollo de la actividad económica. Esto en contraste con un punto bajo, del reactivo P23 (1.8), referido a cómo perciben los profesores una casi nula participación de financiación externa de investigación.

De forma incisiva, vuelve a aparecer la investigación como un elemento trascendente como área de oportunidad para el posgrado, modificando de forma sensible el tema de la actividad económica, ahora se transportan los resultados a la función de fomento, desarrollo y trasmisión de la cultura (ver gráfica 4). 


\section{Gráfica 3. Resultados de la función de fomento y desarrollo de la actividad económica}

Función 3. Fomento y desarrollo de la actividad económica. Puntuación promedio

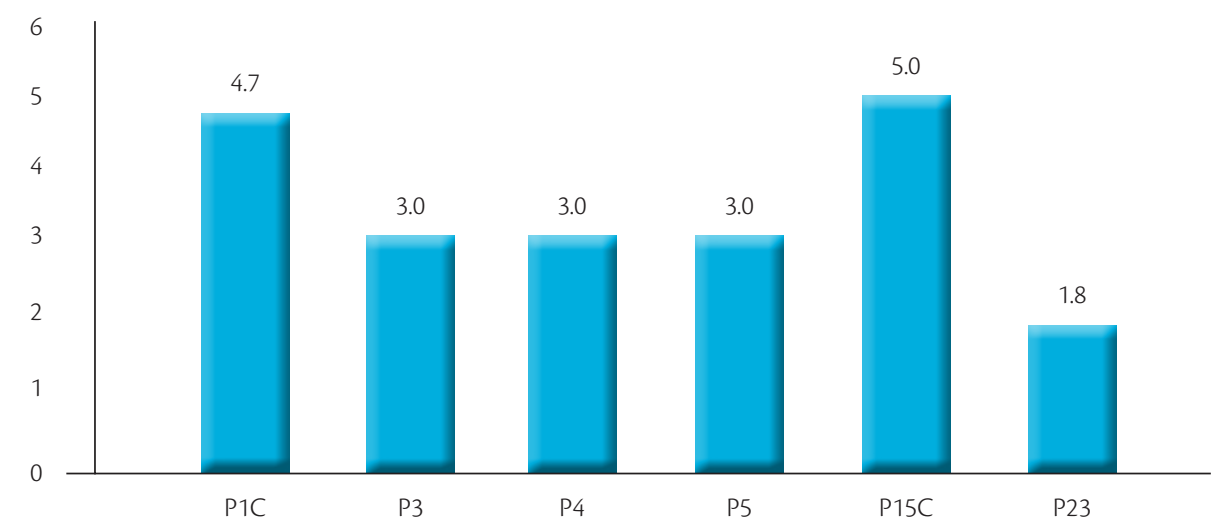

Fuente: elaboración propia de acuerdo con los resultados generados con la investigación.

\section{Gráfica 4. Resultados de la función de fomento, desarrollo y trasmisión de la cultura}

Función 4. Fomento, desarrollo y transmisión de la cultura. Puntuación promedio

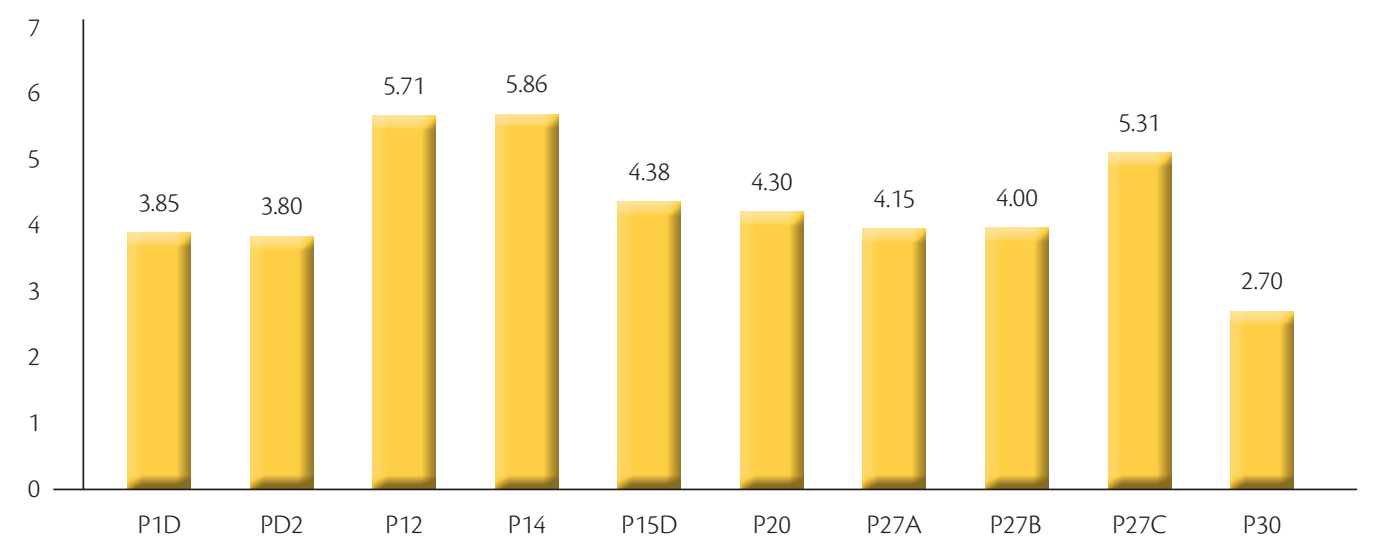

Fuente: elaboración propia de acuerdo con los resultados generados con la investigación.

La función de fomento, desarrollo y trasmisión de la cultura reflejó puntos altos, los cuales pertenecen a las preguntas P14 (5.86), P12 (5.71), P27 (5.31). Nuevamente se cruza la confianza que los maestros tienen en sus métodos de enseñanza, en conjunto con las publicaciones que periódicamente genera el Posgrado en Administración (libros, revistas, ponencias etcétera). En cuanto al punto débil vuelve a relucir P30 (2.7), el concerniente a los intercambios de docentes y posgraduados con otros centros de investigación. Los demás puntos siguen una tendencia de neutral a positiva. 
En otro sentido, la función de la preparación para el mundo profesional, presentó en general una alta valoración en los distintos puntos del instrumento, (gráfica 5), esto obedece a que el Posgrado es de naturaleza profesionalizante, por lo que dicho paradigma favorece esta función del modelo.

Dentro de los puntos sobresalientes en la función preparación para el mundo profesional, se encuentran varios incisos por arriba de los cinco puntos, es decir, es una función privilegiada por el Posgrado. Sobresalieron los reactivos P14 (5.86), P12 (5.71), P22 (5.6), P11 (5.61) y P1E (5.14), resalta que los docentes sienten que están dando las herramientas necesarias en sus respectivas materias y efectuando las evaluaciones para la formación de los futuros maestros en administración.

También sobresalen los principios y valores, y en general la percepción de la profesionalización es buena. De los pocos puntajes bajos, o no tan altos, fueron los puntos P2D (3.31), P8 (4.06) y P29 (4.8). Estos reflejan los intercambios, actividades paralelas a la preparación profesional y la percepción de la satisfacción que tienen los titulados con su formación.

Por último, los resultados y su análisis llegan a la función del compromiso social. Cabe señalar que este aspecto está ligado al entorno, y por ende, es uno de los principales reflejos de la pertinencia social del posgrado.

En general, al igual que las demás funciones, en cuanto al compromiso con la sociedad se manifiestan puntos interesantes, tanto positivos, como con áreas de oportunidad (ver gráfica 6). Esto permite al programa autoevaluarse y replantear sus estrategias para que, de acuerdo con el modelo de funcionalidad empleado, el posgrado pueda mejorar en el sentido de la eficacia organizacional.

\section{Gráfica 5. Resultados de la función preparación para el mundo profesional}

\section{Función 5. Preparación para el mundo profesional. Puntuación promedio}

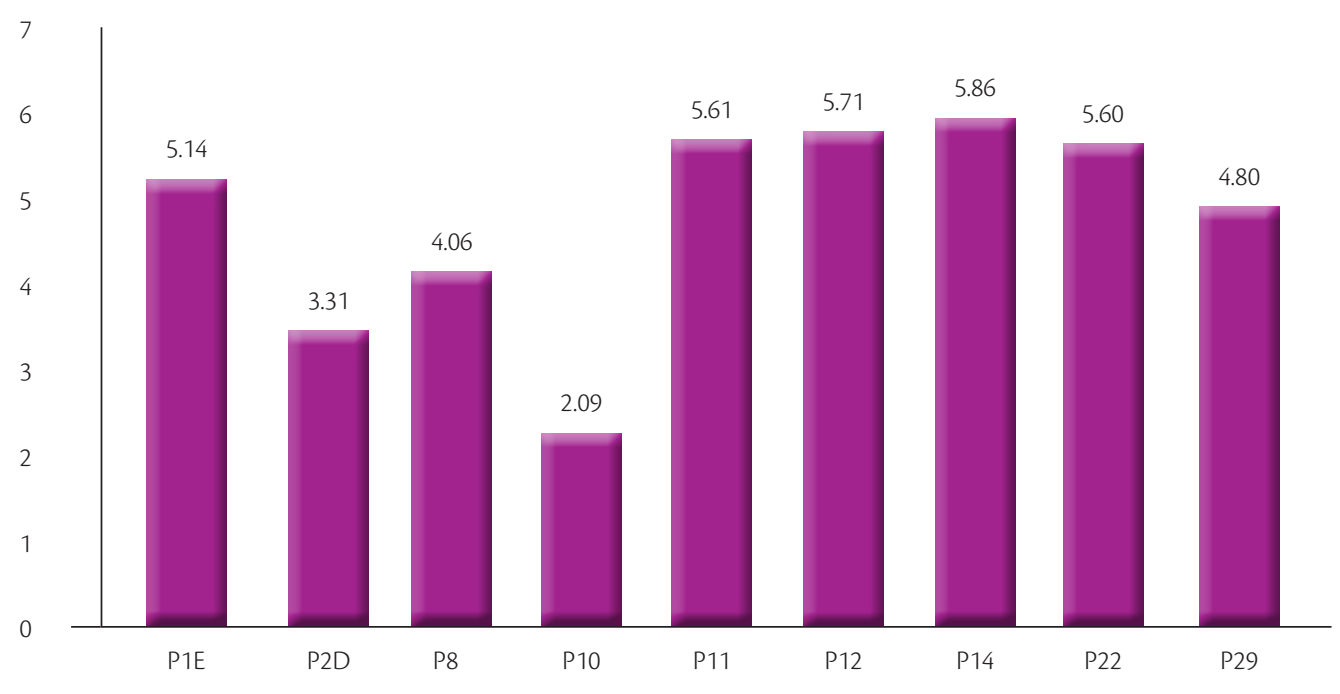


La función del compromiso social en el Posgrado de Administración de la UNISON develó los siguientes aspectos como los de mayor valoración por parte de los profesores: cuestionamientos P22 (5.6), P7B (4.8), P7A (4.38) y P15E (4.38).

De nueva cuenta se muestran los valores de los titulados en primer sitio, seguidos de las metas del posgrado y la atención a las demandas sociales, así como la participación de posgraduados en programas de la maestría. Por lo anterior, puede señalarse que el posgrado es pertinente socialmente en estos aspectos, y con ello corresponde, de acuerdo con la percepción de los profesores, a diversas demandas sociales.

Dentro de los puntos más bajos aparecen las interrogantes P32 (2.5), P31 (2.5), P10 (2.9) y P20 (3.1), compuestas por la respuesta directa de si el posgrado realiza actividades sociales; su cobertura fue calificada negativamente, al igual que los intercambios de los docentes y la presencia del posgrado con trabajos comunitarios es realmente poca, a consideración de la planta docente.

\section{Gráfica 6. Resultados de la función compromiso social}

Función 6. Compromiso social. Puntuación promedio

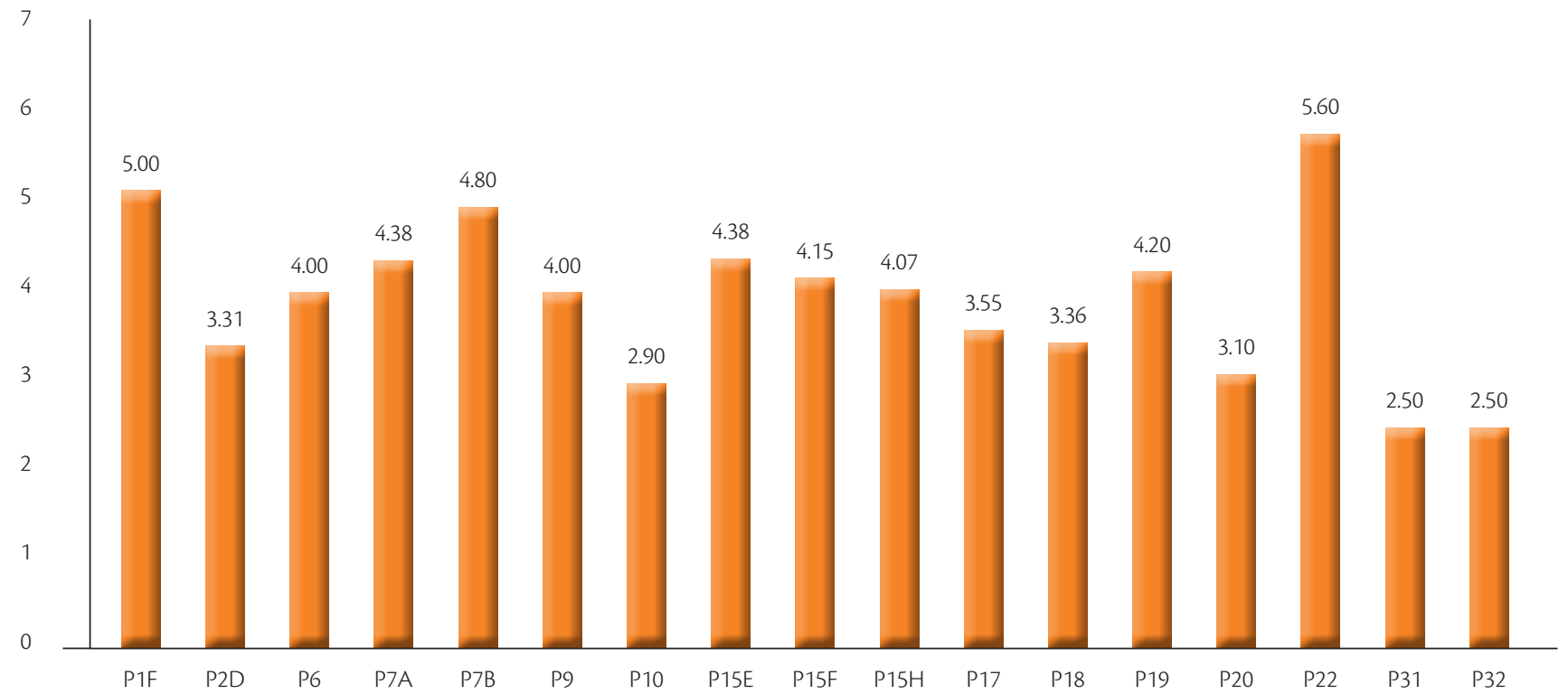

Fuente: elaboración propia de acuerdo con los resultados generados con la investigación. 
Lo anterior implica que existen aún ciertas áreas de mejora para acrecentar el grado de pertinencia social del posgrado, y hacerlo aún más eficaz de lo que ya manifiesta ser, sobre todo hacia el entorno.

De esta manera es como se sobrellevó el análisis particular de cada una de las funciones del modelo, de acuerdo con los resultados de la investigación. Así, a continuación se puede brindar una perspectiva global de la funcionalidad y la eficacia del posgrado.
Los resultados globales del estudio comprenden una vista de la valoración de cada una de las funciones, pero de manera conjunta, y otra correspondiente a un solo cuerpo que representa el grado de eficacia global de las funciones del Posgrado analizado. En la gráfica 7 se muestran los resultados globales de todas y cada una de las funciones del modelo analizado.

\section{Gráfica 7. Valoración de las funciones del Modelo de De la Orden (2007) desde la percepción de los profesores del Posgrado en Administración de la UNIson (2017)}

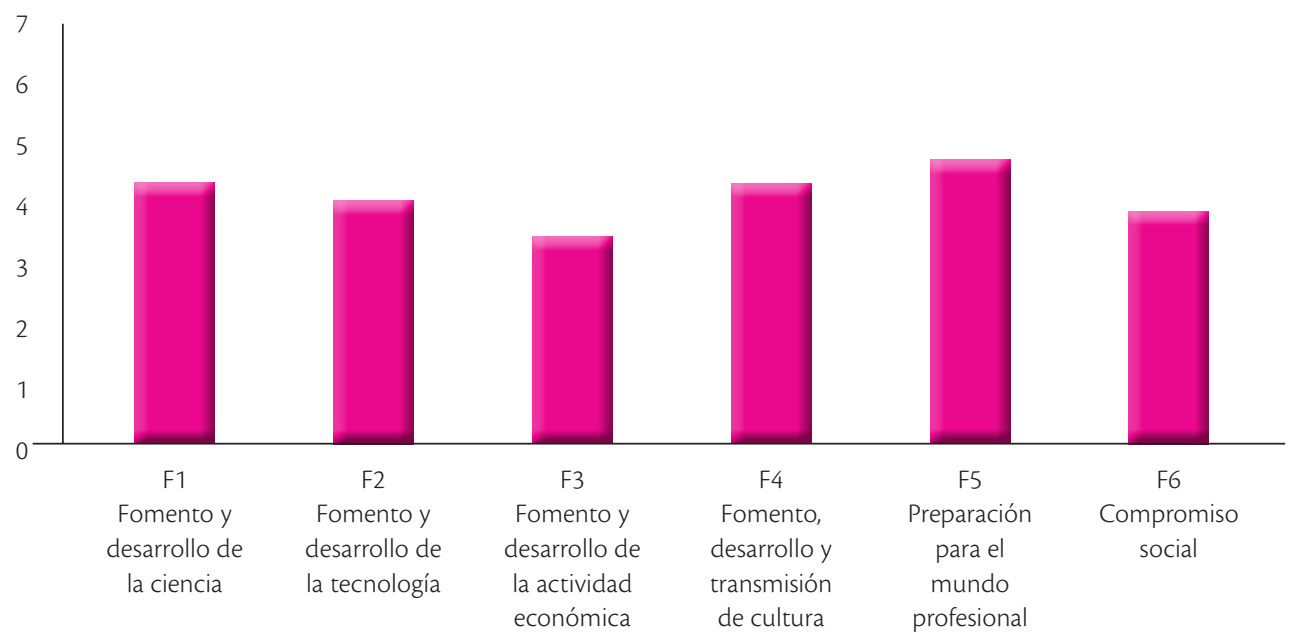

Fuente: elaboración propia de acuerdo con los resultados generados con la investigación.

Cabe recordar que este estudio se basó en la precepción de la planta docente del Posgrado, y que sus percepciones se ajustan al instrumento emanado del modelo de De la Orden (2007). Con ello se pretendió medir la eficacia, pero solamente en las funciones señaladas, por lo que, tras haber censado a la totalidad de los maestros (incluyendo tiempo completo y horas sueltas) se recolectaron 13 instrumentos para esta investigación, y tomando como 7 al punto más alto y 1 más bajo en la escala de Likert, se obtuvo una fluctuación en las diversas funciones universitarias.
En cuanto a la función 1, concerniente al desarrollo de la ciencia, dado que éste no es un posgrado científico sino más tendiente a profesionalización, los docentes otorgaron un promedio de $4.41 / 7$ puntos. Si tomamos 3.5 como punto neutral, podemos decir que se sitúa sólo un poco arriba del promedio. La función 2, fomento y desarrollo tecnológico, tiene 4.03/7 puntos estableciéndose en una zona totalmente neutral en el estudio. La función número 3, desarrollo de la actividad económica, que por la naturaleza de la Maestría se esperaría con una alta puntuación, sólo obtuvo $3.41 / 7$, por debajo del punto medio de la escala de Likert. 
Por otra parte, la función 4 resultó con 4.406, casi a la par de la función número uno, que no es tan cercana al posgrado que se está estudiando. La función 5, preparación para el mundo profesional, de la que también se esperaría un alto puntaje, arroja un promedio de $4.81 / 7$, casi 5 puntos, y es la de mayor valoración en el estudio. En la última función del instrumento, compromiso social, los maestros del posgrado la catalogaron con 3.81/7, un puntaje algo bajo.

Más adelante, se presentan los valores porcentuales globales del estudio, en la tabla 3 y la gráfica 8 .

De acuerdo con el panorama de los resultados, en términos de porcentajes, se puede observar (gráfica
8) que la función de preparación para el mundo profesional ocupa el primer lugar con el $68.77 \%$ de cumplimiento de eficacia en ese sector, seguido de la función de fomento y desarrollo de la ciencia, con un $63.10 \%$. El tercer lugar lo ocupa la función de fomento, desarrollo y trasmisión de la cultura, con el $62.95 \%$; en cuarto lugar aparece la función de fomento y desarrollo de la tecnología, con $57.53 \%$; en quinto sitio la función de compromiso social, con un $55.30 \%$ de eficacia, y por último, la función de fomento y desarrollo de la tecnología con el $48.81 \%$, siendo la menos eficaz, según los resultados de la investigación, que reflejan la percepción de los maestros del Posgrado en Administración de la Universidad de Sonora.

Tabla 3. Expresión porcentual de la percepción de los docentes en cuanto a la eficacia de las funciones del modelo

\begin{tabular}{|l|c|c|}
\hline \multicolumn{1}{|c|}{ Tabla de cumplimiento de funciones según docentes } & P/Función & \% Cumplimiento F/7 \\
\hline F1 Fomento y desarrollo de la ciencia & 4.41672962 & $63.10 \%$ \\
\hline F2 Fomento y desarrollo de la tecnología & 4.03375000 & $57.63 \%$ \\
\hline F3 Fomento y desarrollo de la actividad económica & 3.41666667 & $48.81 \%$ \\
\hline F4 Fomento, DESARROLLO Y TRASMISIÓN DE CULTURA & 4.40679487 & $62.95 \%$ \\
\hline F5 Preparación para el mundo profesional & 4.81416524 & \\
\hline F6 Compromiso social & 3.87104072 & $68.77 \%$ \\
\hline
\end{tabular}

Fuente: elaboración propia de acuerdo con los resultados generados con la investigación. 


\section{Gráfica 8. Cumplimiento global porcentual de las funciones del modelo de funcionalidad de acuerdo con la percepción de los docentes}

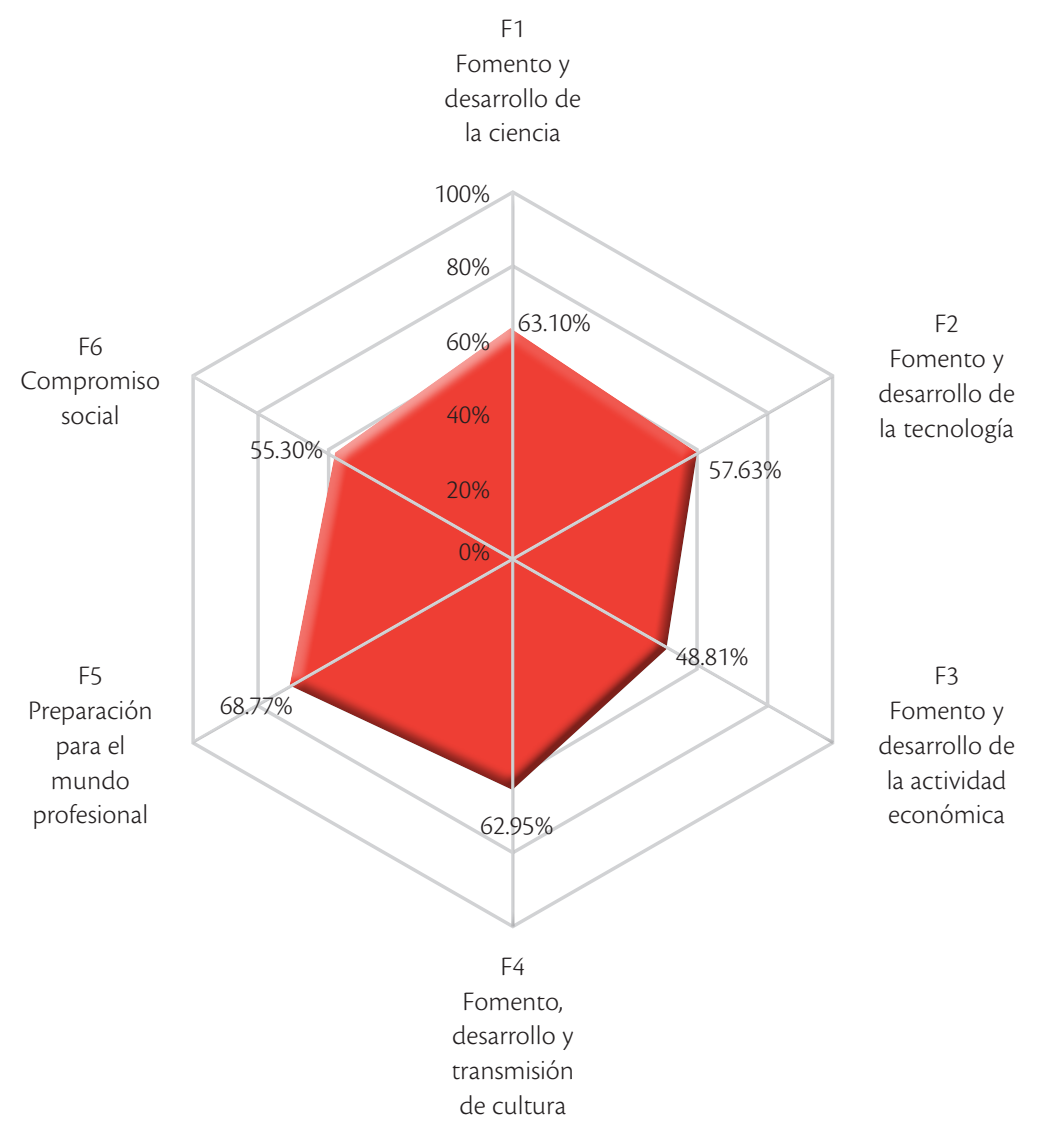

Fuente: elaboración propia de acuerdo con los resultados generados con la investigación.

\section{Conclusiones}

Para iniciar con la conclusión, se pueden tomar los tres primeros lugares de las funciones de la Maestría en Administración de la Universidad de Sonora como sus fortalezas, sin embargo, aún se tienen áreas de oportunidad, pues probablemente, para ser considerado como nivel óptimo esperado, habría que contar con una valoración de un $80 \%$ a un $90 \%$. Por lo tanto, aunque el modelo utilizado en el estudio, y la naturaleza del programa de Posgrado difieran, es necesario reflexionar si las funciones que se realizan son las necesarias y en qué grado se logran cumplir de manera eficaz.
De igual manera, la función de preparación para el mundo profesional, percibida por los docentes como la más eficaz, con un $68.77 \%$ (equivalente a $4.814 / 7$ ), requiere un mayor fortalecimiento. Es positivo que sea un punto fuerte, sin embargo es preocupante que tenga una valoración casi equivalente a la función del desarrollo de la ciencia, con una diferencia de sólo 5 puntos porcentuales, considerando que la ciencia no es el objeto principal de la Maestría, pero preparar profesionalmente es preponderante.

El posgrado en Administración acaba de pasar por una transición importante, con un cambio de programa después de 37 años de vida en la institución. 
Aun así, los maestros ven una carencia que no está siendo cubierta; los docentes tienen una marcada confianza en las materias que imparten, en lo particular, pero ven un área de oportunidad importante.

De la misma forma, se cuenta con una sólida trasmisión del conocimiento, así como con publicaciones que los maestros emiten periódicamente. Sin embargo, esto no es del conocimiento de la totalidad de maestros (horas sueltas), por ello la puntuación se ve afectada. También las ponencias de los profesores son altamente reconocidas, algunas a nivel internacional.

Ante la dinámica actual, se requieren intercambios para la investigación en otras partes del mundo, para enriquecer la cultura del posgrado en cuestión y la manera de proceder de otras instituciones, e integrar lo más conveniente a la propia. Incluso se pudiera aprovechar el contacto que hay con los posgraduados para este mismo tema.

Por otra parte, la Maestría en Administración se complementa con el ámbito social porque simplemente no se encuentra aislada, es parte de una comunidad que espera de ella ciertos estándares. Vemos un marcador bastante bajo a los ojos de los docentes en cuanto a las acciones de la función del compromiso social, por lo que debiera promoverse y conjuntar los recursos para detonar esta función en el Posgrado.

Los programas de investigación en campo empresarial pudieran ayudar, mediante la firma de un mayor número de convenios con las empresas, en los cuales éstas puedan manifestar sus necesidades y ver cómo implementarlas en el programa. Aparte de los trabajos finales de los alumnos que se implementan en empresas, sería muy importante que el posgrado tuviese un mayor acercamiento con las empresas, para que los estudiantes realicen estudios y metanoias, ya que en realidad existen algunas barreras para realizarlos. Es una dificultad importante que los alumnos encuentran hoy en día para realizar cualquier tipo de estudio.
En definitiva, falta generar una cultura de apertura para la investigación y el conocimiento, tanto en los estudiantes como en las empresas. Con la existencia de convenios entre empresas y posgrado, tanto docentes como alumnos pueden tener acceso y facilitar los estudios y saber cuáles son las necesidades de diversos tipos de empresas para focalizar y enriquecer sus aportaciones; todo esto en el mundo profesional, para lograr una formación pertinente para afrontar diversas situaciones y problemas. Parte de ese convenio podría ser una bolsa de trabajo para ver que la empresa se beneficie con capital humano confiable recomendado por el Posgrado de Administración, en un convenio ganar-ganar entre empresarios y posgrado. Los estudiantes y docentes tendrían acceso a la información y practica de proyectos sin costo para las empresas y potencial capital humano muy competente.

Si hablamos de transmisión de cultura, es cierto que los académicos viajan a presentar trabajos y ponencias en congresos de diversas localidades, incluso internacionales, pero se carece de un programa de intercambio para docentes para enriquecer al programa y motivarlos, lo que generaría una evolución más acelerada del posgrado y estar al tanto de los últimos avances en materias de interés y diferentes maneras de implementar e impartir conocimiento.

Ahora bien, en el ámbito social, como ya se mencionó la maestría no está aislada, se inserta en una comunidad donde se desenvuelve y se esperan ciertas cualidades de ella, y que destaque en cuanto a lo social. De nuevo aparece la idea de los convenios con empresarios, bolsa de trabajo ejecutiva, acercamientos a instituciones sin fines de lucro, PYMES, empresas familiares y ligar el compromiso con la responsabilidad social. En particular, convenios con empresarios para facilitar el acceso de los alumnos y docentes a la información y trabajos para las mismas empresas que puedan verse beneficiados con informes y diagnósticos que pudieran generar ingresos. También se puede participar en una bolsa de trabajo, 
así los empresarios tienen la seguridad de contar con una base de datos confiable y de calidad; mientras que los alumnos pueden tener la opción de mejorar sus empleos actuales u obtener uno nuevo. Aunque ya se firmaron convenios para la restructuración del programa en 2010, aún existe una barrera fuerte para que los estudiantes puedan realizar estudios de la Maestría en Administración.

En este proceso de vinculación, podría ser importante incluir instituciones sin fines de lucro. Por ejemplo, se pueden generar estrategias de gestión de recursos para el mantenimiento de un orfanato o un banco de alimentos. La presencia del posgrado elevaría la imagen de éstos notablemente, haciendo que la sociedad vea mejores expectativas y una imagen sólida de la Maestría en Administración y de la Universidad de Sonora.

$\mathrm{El}$ acercamiento a las PYMES y empresas familiares, que son bastantes, puede generar espacios para el involucramiento en su administración, es muy especial cuando relacionamos al núcleo social más importante que es la familia, con el núcleo económico más importante, la empresa. Esto puede hacer que los estudiantes se sientan más preparados a emprender un negocio propio o establecer tablas más firmes en caso de ya contar con uno de estos negocios. También las pequeñas empresas se verían beneficiadas con estudios que en corto o mediano plazo no pudieran costear, pero sí serían de utilidad, y los estudiantes y docentes tendrían un mejor panorama en ese sector del cual no se tiene gran información.

Por último, debe comentarse que un posgrado en administración que se complementa con lo mencionado, en aspectos importantes como el compromiso social y la preparación para el mundo profesional, con el tiempo puede generar mejores resultados en materia de eficacia. Así pues, las demás funciones van entrelazadas para formar un conjunto funcional y armónico en el cual la Maestría en Administración presenta áreas de oportunidad, y las cuales sería interesante desarrollar para lograr un posgrado saludable, eficaz y funcional. Quedará como reto la posibilidad de aplicar un análisis de corte paramétrico con la finalidad de propiciar una valoración desde otro enfoque, además de incluir a los demás actores que participan en el proceso formativo, y con ello generar mayores aportaciones al tema y al ámbito de la gestión de la universidad y la educación superior. 


\section{Referencias}

Claude, G. et al. (2005), Historia del pensamiento administrativo ( $2^{\mathrm{a}}$ ed.), México, Pearson Education.

Chiavenato, I. (2014), Introducción a la teoría general de la administración, México, McGraw Hill.

Coronado, G. M. (2017), Pertinencia social universitaria, enfoques y perspectivas de profesores estudiantes y empleadores, Hermosillo, México, Editorial Qartuppi, DOI: 10.29410/QTP.17.03.

De la Orden, A. et al. (2007), "Niveles y perfiles de funcionalidad como dimensión de calidad universitaria. Un estudio empírico en la Universidad Complutense", Archivos Analíticos de Políticas Educativas, 15 (12), <http:/ / epaa.asu.edu/epaa/> [Consulta: noviembre de 2018].

Didrikson, A. (2008), "Contexto global y regional de la educación superior en América Latina y el Caribe", en A. L. Gazzola y A. Didriksson (eds.), Tendencias de la Educación Superior en América Latina y el Caribe, Caracas, UNESCO, pp. 1-43.
Fisher S., Dornbush, R. y Schmalensee, R. (2004) Macroeconomía, Décima Edición, México, McGraw Hill. García, G. J. (2009), "Las políticas y los programas de posgrado", Sociológica, año 24, número 70, mayoagosto, pp. 153-174.

Koontz, H. y H. Weihrich, H. (2004), Administración: Una perspectiva global, México, McGraw Hill.

OCDE (2005), Estudios Económicos de la OCDE, México, disponible en: <https://www.gob.mx/cms/uploads/ attachment/file/483576/Estudios_Economicos_de_ la_OCDE_Mexico_2019.pdf> [Consulta: agosto de 2018].

Robbins, S. P. y M. Coulter (2005), Administración, México, Pearson Education.

Valenzuela, J. yJ. Ramirez (2011), “Cultura de evaluación en instituciones educativas", Perfiles Educativos, vol. XXXIII, núm. 131, pp 42-63.

\section{Cómo citar este artículo:}

Coronado-García, Manuel-Arturo, Sergio-Ramón Rossetti-López e Isaac-Shamir Rojas-Rodríguez (2020), "Funcionalidad y eficacia del Posgrado. La visión del profesorado en la Universidad de Sonora", Revista Iberoamericana de Educación Superior (RIES), vol. XI, núm. 32, pp. 67-86, DOI: https://doi.org/10.22201/iisue.20072872e.2020.32.816 [Consulta: fecha de última consulta]. 